\title{
Next-to-leading order QCD predictions for dijet photoproduction in lepton-nucleus scattering at the future EIC and at possible LHeC, HE-LHeC, and FCC facilities
}

\author{
V. Guzey $\oplus^{1}$ and M. Klasen $\oplus^{2}$ \\ ${ }^{1}$ National Research Center “Kurchatov Institute,” Petersburg Nuclear Physics Institute (PNPI), Gatchina, 188300, Russia \\ ${ }^{2}$ Institut für Theoretische Physik, Westfälische Wilhelms-Universität Münster, Wilhelm-Klemm-Straße 9, 48149 Münster, Germany
}

(Received 24 March 2020; revised 1 October 2020; accepted 18 November 2020; published 4 December 2020)

\begin{abstract}
We calculate cross sections for inclusive dijet photoproduction in electron-nucleus scattering in the kinematics of the future EIC and the possible $\mathrm{LHeC}$, HE-LHeC, and FCC using next-to-leading order (NLO) perturbative QCD and nCTEQ15 and EPPS16 nuclear parton density functions (nPDFs). We make predictions for distributions in the dijet average transverse momentum $\bar{p}_{T}$, the average rapidity $\bar{\eta}$, the observed nuclear momentum fraction $x_{A}^{\mathrm{obs}}$, and the observed photon momentum fraction $x_{\gamma}^{\mathrm{obs}}$. Comparing the kinematic reaches of the four colliders, we find that an increase of the collision energy from the EIC to the LHeC and beyond extends the coverage in all four considered variables. Notably, the LHeC and HE-LHeC will allow one to probe the dijet cross section down to $x_{A}^{\mathrm{obs}} \sim 10^{-4}$ (down to $x_{A}^{\mathrm{obs}} \sim 10^{-5}$ at the FCC). The ratio of the dijet cross sections on a nucleus and the proton, $\sigma_{A} /\left(A \sigma_{p}\right)$, depends on $x_{A}^{\text {obs }}$ in a similar way as the ratio of gluon densities, $g_{A}\left(x_{A}, \mu^{2}\right) /\left[A g_{p}\left(x_{A}, \mu^{2}\right)\right]$, for which current nPDFs predict a strong suppression due to nuclear shadowing in the region $x_{A}^{\text {obs }}<0.01$. Dijet photoproduction at future lepton-nucleus colliders can therefore be used to test this prediction and considerably reduce the current uncertainties of nPDFs.
\end{abstract}

DOI: 10.1103/PhysRevC.102.065201

\section{INTRODUCTION}

Lepton-nucleus scattering at high energies has traditionally been a fruitful way to access and study the structure of nuclei in quantum chromodynamics (QCD). Despite numerous successes and insights, there is an overarching need to continue these studies at progressively higher energies using colliders. While the plans to use nuclear beams in the HERA collider at DESY [1] have not materialized, a high-energy polarized lepton-proton and lepton-nucleus collider at Brookhaven National Laboratory (BNL) [2,3] —an Electron-Ion Collider (EIC) - has recently been approved. Further down the road, one envisions that the Large Hadron Collider (LHC) at CERN will be complemented by a Large Hadron-Electron Collider $(\mathrm{LHeC})$ and its higher energy upgrade (HE-LHeC) $[4,5]$ as well as a future circular collider (FCC) [6].

The core of the physics program at the future leptonnucleus colliders is comprised of deep inelastic scattering (DIS), allowing one to map out various parton distributions in nuclei with high precision; see, e.g., Refs. [7-10]. In addition, as one learned from HERA, photoproduction of jets $[11,12]$ and dijets $[13,14]$ provides useful complementary information on the QCD (and in particular gluon) structure of hadrons. This has recently been exploited at the LHC, where ultrape-

Published by the American Physical Society under the terms of the Creative Commons Attribution 4.0 International license. Further distribution of this work must maintain attribution to the author(s) and the published article's title, journal citation, and DOI. Funded by $S C O A P^{3}$. ripheral collisions (UPCs) of heavy ions give an opportunity to study photon-nucleus scattering at unprecedentedly high energies [15]. In particular, it was shown that inclusive dijet photoproduction in $\mathrm{Pb}-\mathrm{Pb}$ UPCs at the LHC can help to reduce the existing uncertainty in nuclear parton distribution functions (nPDFs) at small $x$ by approximately a factor of 2 $[16,17]$.

In this work, we calculate the cross section of inclusive dijet photoproduction in electron-nucleus scattering in the kinematics of the future $\mathrm{EIC}, \mathrm{LHeC}, \mathrm{HE}-\mathrm{LHeC}$, and FCC using the formalism of collinear factorization, nextto-leading order (NLO) perturbative QCD, and nCTEQ15 [18] and EPPS16 [19] nPDFs. We make predictions for the cross-section distributions as functions of the dijet average transverse momentum $\bar{p}_{T}$, the average rapidity $\bar{\eta}$, the observed nuclear momentum fraction $x_{A}^{\text {obs }}$, and the observed photon momentum fraction $x_{\gamma}^{\text {obs }}$. We compare the kinematic reaches of the four colliders and find that an increase of the collision energy from the EIC to the $\mathrm{LHeC}$ and beyond extends the coverage in all four considered variables. Notably, the $\mathrm{LHeC}$ and $\mathrm{HE}-\mathrm{LHeC}$ will allow one to probe the dijet cross section down to $x_{A}^{\text {obs }} \sim 10^{-4}$ (down to $x_{A}^{\text {obs }} \sim 10^{-5}$ at the FCC), which is two (three) orders of magnitude smaller than that at the EIC. We then discuss in detail the implications of future measurements of dijet photoproduction in lepton-nucleus scattering on the determination of nPDFs.

This work continues and extends the analysis of Ref. [20] by making predictions for high-energy lepton-nucleus colliders including $\mathrm{LHeC}$, HE-LHeC, and FCC, comparing them to the case of the EIC, and analyzing relative merits of the four considered colliders. 
TABLE I. Energy configurations of electron-ion colliders considered in this work.

\begin{tabular}{lccc}
\hline \hline & $E_{e}(\mathrm{GeV})$ & $E_{A}(\mathrm{TeV})$ & $\sqrt{s}(\mathrm{GeV})$ \\
\hline EIC & 21 & 0.1 & 92 \\
LHeC & 60 & 2.76 & 812 \\
HE-LHeC & 60 & 4.93 & 1,088 \\
FCC & 60 & 19.7 & 2,174 \\
\hline \hline
\end{tabular}

The remainder of the paper is structured as follows. In Sec. II, we recap the formalism and the input for the calculation of inclusive dijet photoproduction in NLO perturbative QCD. Our results and their discussion are presented in Sec. III. A summary of our results is given in Sec. IV.

\section{DIJET PHOTOPRODUCTION IN NEXT-TO-LEADING ORDER QCD}

In the framework of collinear factorization and next-toleading order (NLO) perturbative QCD [21-25], the cross section of dijet photoproduction in $e A \rightarrow e+2$ jets $+X$
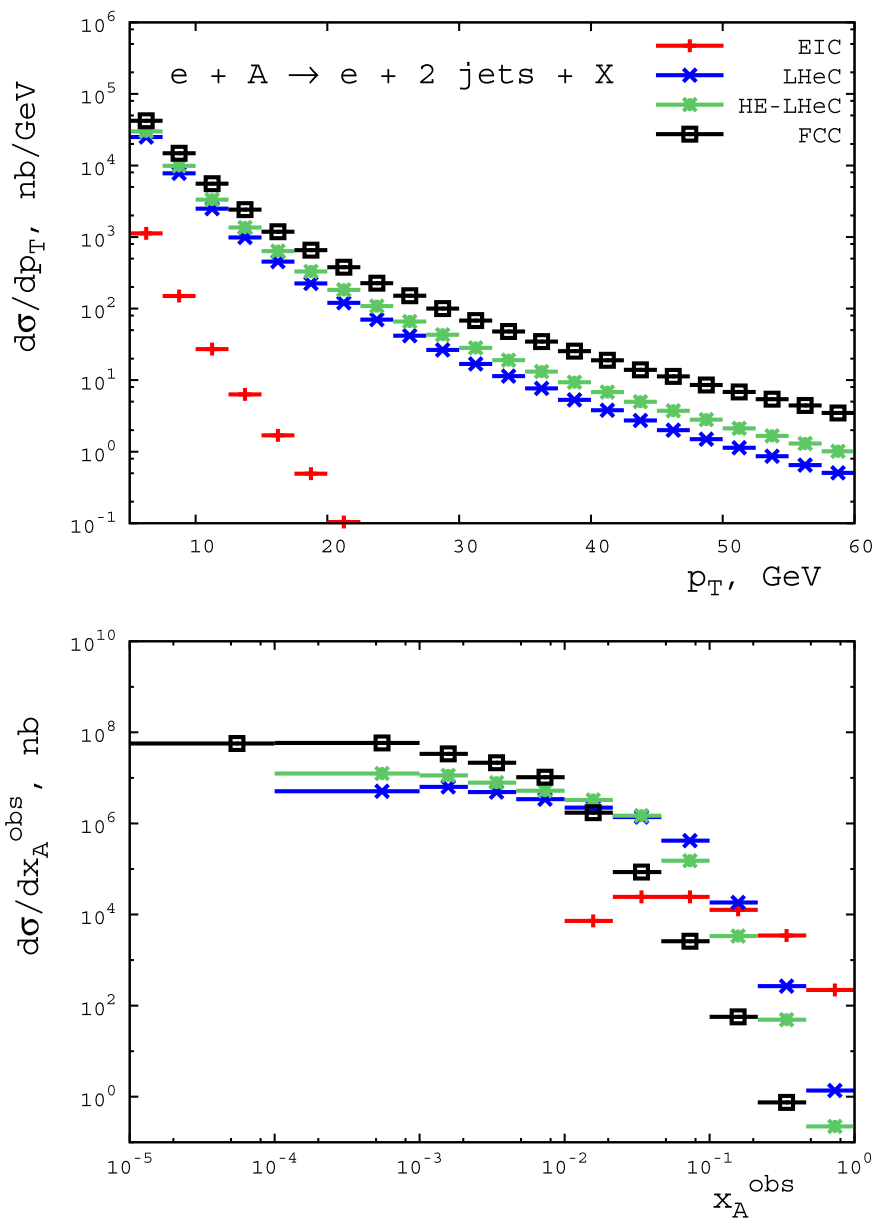

electron-nucleus scattering reads

$$
\begin{aligned}
& d \sigma(e A \rightarrow e+2 \text { jets }+X) \\
& =\sum_{a, b} \int d y \int d x_{\gamma} \int d x_{A} f_{\gamma / e}(y) f_{a / \gamma}\left(x_{\gamma}, \mu^{2}\right) \\
& \quad \times f_{b / B}\left(x_{A}, \mu^{2}\right) d \hat{\sigma}(a b \rightarrow \text { jets }),
\end{aligned}
$$

where $a, b$ are parton flavors; $f_{\gamma / e}(y)$ is the flux of equivalent photons of the electron, which depends on the photon light-cone momentum fraction $y ; f_{a / \gamma}\left(x_{\gamma}, \mu^{2}\right)$ is the PDF of the photon for the resolved photon case (see below), which depends on the momentum fraction $x_{\gamma}$ and the factorization scale $\mu ; f_{b / B}\left(x_{A}, \mu^{2}\right)$ is the nuclear PDF with $x_{A}$ being the corresponding parton momentum fraction; and $d \hat{\sigma}(a b \rightarrow$ jets $)$ is the elementary cross section for the production of two-parton and three-parton final states emerging as jets in hard scattering of partons $a$ and $b$.

The dijet cross section in Eq. (1) receives two types of contributions: the resolved photon contribution, when the photon interacts with target partons through its quark-gluon structure expressed by $f_{a / \gamma}\left(x_{\gamma}, \mu^{2}\right)$, and the direct photon contribution, when the photon enters directly the hard scattering cross section $d \hat{\sigma}(a b \rightarrow$ jets $)$. At leading order (LO), the direct
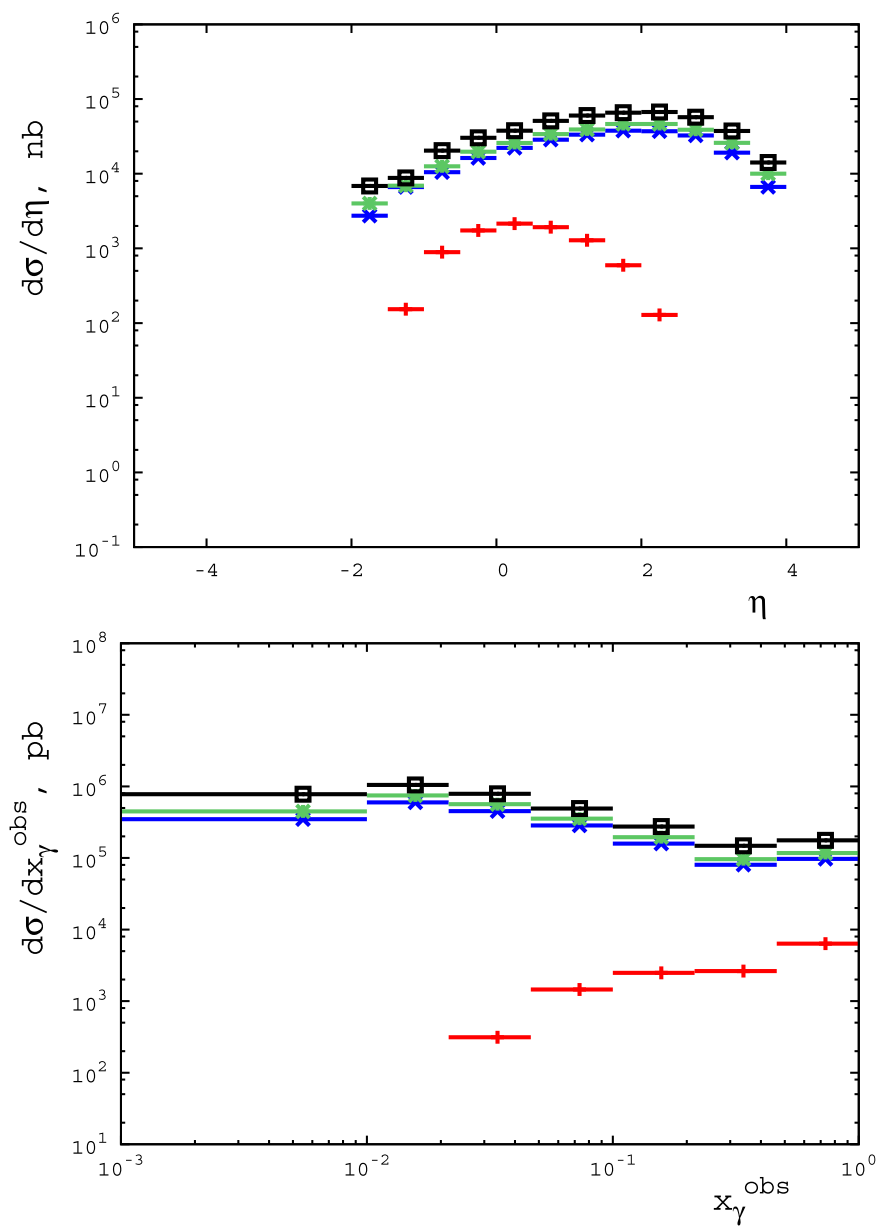

FIG. 1. NLO QCD predictions for the dijet photoproduction cross section in $e A \rightarrow e+2$ jets $+X$ electron-nucleus scattering at the EIC, $\mathrm{LHeC}, \mathrm{HE}-\mathrm{LHeC}$, and FCC as a function of the average dijet transverse momentum $\bar{p}_{T}$, the average rapidity $\bar{\eta}$, and the momentum fractions $x_{A}^{\mathrm{obs}}$ and $x_{\gamma}^{\mathrm{obs}}$. The calculation uses nCTEQ15 nPDFs. 

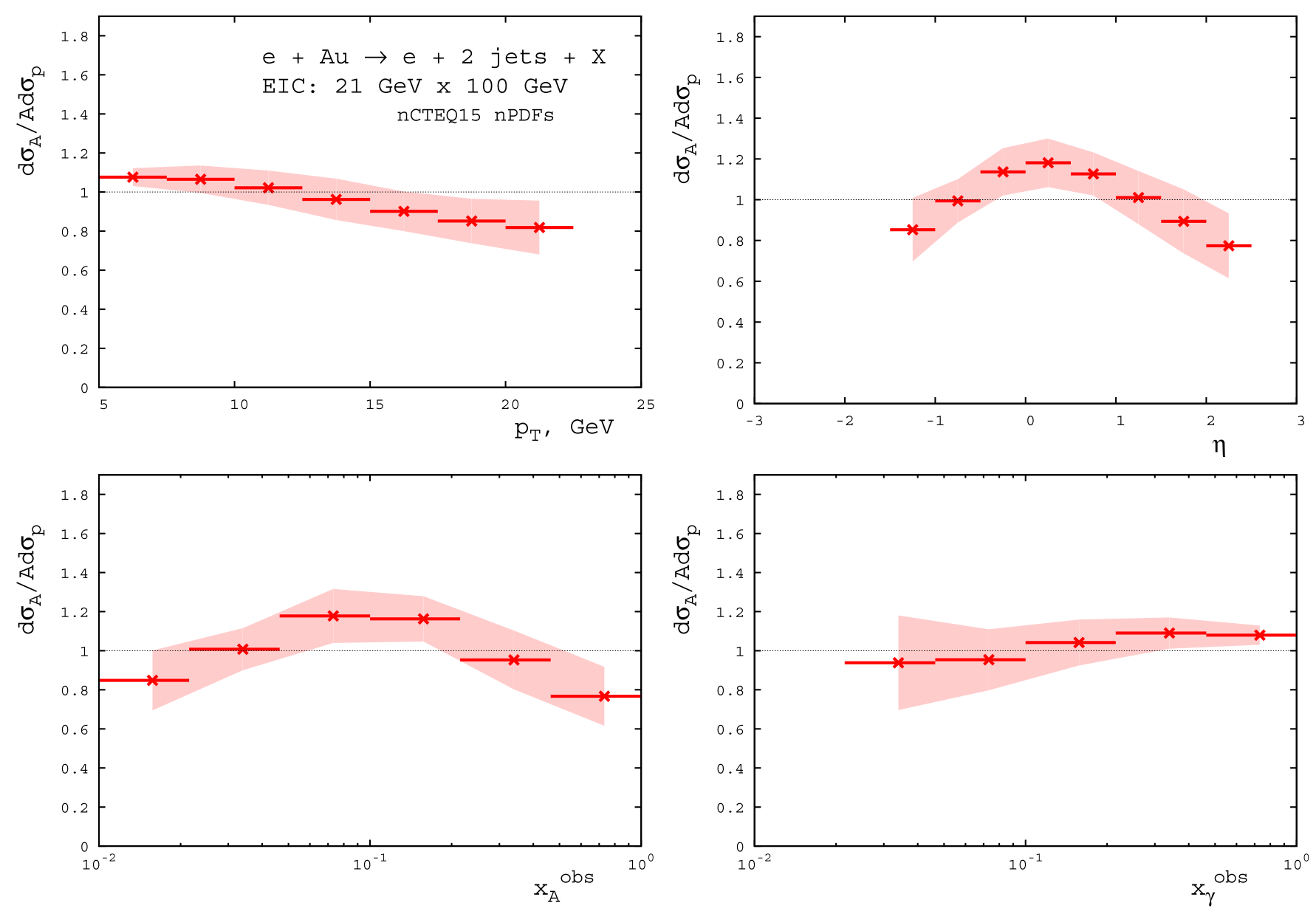

FIG. 2. NLO QCD predictions for the ratio of the cross sections of dijet photoproduction on nuclei and the proton as a function of $\bar{p}_{T}$, $\bar{\eta}$, $x_{A}^{\mathrm{obs}}$, and $x_{\gamma}^{\mathrm{obs}}$ in the EIC kinematics. The calculation uses central values of nCTEQ15 nPDFs (solid lines) and 32 sets of error PDFs (shaded band).

photon contribution has the support exactly at $x_{\gamma}=1$ and $f_{\gamma / \gamma}\left(x_{\gamma}, \mu^{2}\right)=\delta\left(1-x_{\gamma}\right)$. At NLO, the separation between the resolved and direct photon contributions depends on the factorization scheme and scale $\mu$. Indeed, by calculating the virtual and real corrections to the matrix elements of interest using massless quarks in dimensional regularization, one can explicitly show that ultraviolet (UV) divergences are renormalized in the $\overline{\mathrm{MS}}$ scheme and infrared (IR) divergences are canceled and factorized into the nucleus (proton) and photon PDFs, respectively; see Ref. [25]. For the latter, this can imply a transformation from the DIS $\gamma$ to the $\overline{\mathrm{MS}}$ scheme. As a result, the direct photon contribution becomes sizable and in practice dominates the cross section at $x_{\gamma} \approx 1$ even at NLO.

In our analysis, we used for the photon flux of the electron the improved expression derived in the Weizsäcker-Williams approximation [26]

$$
\begin{aligned}
f_{\gamma / e}(y)= & \frac{\alpha}{2 \pi}\left[\frac{1+(1-y)^{2}}{y} \ln \frac{Q_{\max }^{2}(1-y)}{m_{e}^{2} y^{2}}\right. \\
& \left.+2 m_{e}^{2} y\left(\frac{1}{Q_{\max }^{2}}-\frac{1-y}{m_{e}^{2} y^{2}}\right)\right],
\end{aligned}
$$

where $\alpha$ is the fine-structure constant; $m_{e}$ is the electron mass; and $Q_{\max }^{2}$ is the maximal photon virtuality. Motivated by studies of jet photoproduction at HERA, we take $Q_{\max }^{2}=$ $0.1 \mathrm{GeV}^{2}$ and assume that the inelasticity spans the range of $0<y<1$.

For the photon PDFs, we used the GRV HO parametrization [27], which we transformed as explained above. These photon PDFs have been tested thoroughly at HERA and the Large Electron Positron (LEP) collider at CERN and are very robust, especially at high $x_{\gamma}$ (dominated by the pQCD photonquark splitting), which is correlated with the low- $x_{A}$ region that is of particular interest for this work. For the nuclear PDFs $f_{b / B}\left(x_{A}, \mu^{2}\right)$, we employed the nCTEQ15 [18] and EPPS16 [19] parametrizations including both central and error PDFs. The latter are used to evaluate the theoretical uncertainty bands of our predictions.

\section{PREDICTIONS FOR DIJET PHOTOPRODUCTION CROSS SECTIONS AT FUTURE ELECTRON-ION COLLIDERS}

We performed perturbative NLO QCD calculations of the dijet photoproduction cross section using Eq. (1), which was numerically implemented in an NLO parton-level Monte Carlo [21-25]. This framework has been successfully tested 
to describe the HERA and LEP data on dijet photoproduction on the proton. It implements the anti- $k_{T}$ algorithm [28] with a jet radius of $R=0.4$ (we have at most two partons in the jet) and the following generic conditions on final-state jets: The leading jet has $p_{T, 1}>5 \mathrm{GeV}$, while the other jets have a lower cut on $p_{T, i \neq 1}>4.5 \mathrm{GeV}$ to avoid an enhanced sensitivity to soft radiation in the calculated cross section [29]; all jets have rapidities $\left|\eta_{1,2}\right|<4$. The studied energy configurations of future electron-ion colliders are summarized in Table I, where $E_{e}$ and $E_{A}$ refer to the electron and nucleus beam energies, respectively, and $\sqrt{s}$ is the center-of-mass collision energy per nucleon.

In general, i.e., beyond leading order (LO) perturbative QCD, the light-cone momentum fractions $x_{\gamma}$ and $x_{A}$ in Eq. (1) are not directly measurable. Instead, one usually introduces their estimates, which can be defined using the two highest transverse-energy jets,

$$
\begin{aligned}
& x_{\gamma}^{\mathrm{obs}}=\frac{p_{T, 1} e^{-\eta_{1}}+p_{T, 2} e^{-\eta_{2}}}{2 y E_{e}}, \\
& x_{A}^{\mathrm{obs}}=\frac{p_{T, 1} e^{\eta_{1}}+p_{T, 2} e^{\eta_{2}}}{2 E_{A}}
\end{aligned}
$$

where $p_{T, 1,2}$ and $\eta_{1,2}$ are the transverse energies and rapidities of the two jets $\left(p_{T, 1}>p_{T, 2}\right)$.

Figure 1 summarizes our predictions for the dijet cross section, Eq. (1), as a function of the dijet average transverse momentum $\bar{p}_{T}=\left(p_{T, 1}+p_{T, 2}\right) / 2$, the average rapidity $\bar{\eta}=\left(\eta_{1}+\eta_{2}\right) / 2$, and the momentum fractions $x_{A}^{\mathrm{obs}}$ and $x_{\gamma}^{\mathrm{obs}}$. The calculations are performed using the central value of the nCTEQ15 nPDFs. On a logarithmic $y$ scale, EPPS16 nPDFs give indistinguishable results. We find sizable yields in all four considered variables. In particular, at the EIC the kinematic coverage spans $5 \leqslant \bar{p}_{T} \leqslant 20 \mathrm{GeV},-2<\bar{\eta} \leqslant 3$, $0.03 \leqslant x_{\gamma}^{\text {obs }} \leqslant 1$, and $0.01 \leqslant x_{A}^{\text {obs }} \leqslant 1$; see also Ref. [20]. Comparing the kinematic reaches of the four colliders, one can see from the figure that an increase of the collision energy dramatically expands the kinematic coverage. At the LHeC, $\mathrm{HE}-\mathrm{LHeC}$, and FCC, one probes the dijet cross section in the wider ranges of $5 \leqslant \bar{p}_{T} \leqslant 60 \mathrm{GeV},-2 \leqslant \bar{\eta} \leqslant 4,10^{-3} \leqslant$ $x_{\gamma}^{\text {obs }} \leqslant 1$, and $10^{-4} \leqslant x_{A}^{\text {obs }} \leqslant 1$ (LHeC and HE-LHeC), and even $10^{-5} \leqslant x_{A}^{\text {obs }} \leqslant 1$ (FCC).

To quantify the magnitude of nuclear modifications of the calculated cross section, we show the ratios of the nuclear cross section, Eq. (1), to the cross section of dijet photoproduction on the proton, $d \sigma_{A} /\left(A d \sigma_{p}\right)$, in Figs. 2 and 3 in the EIC
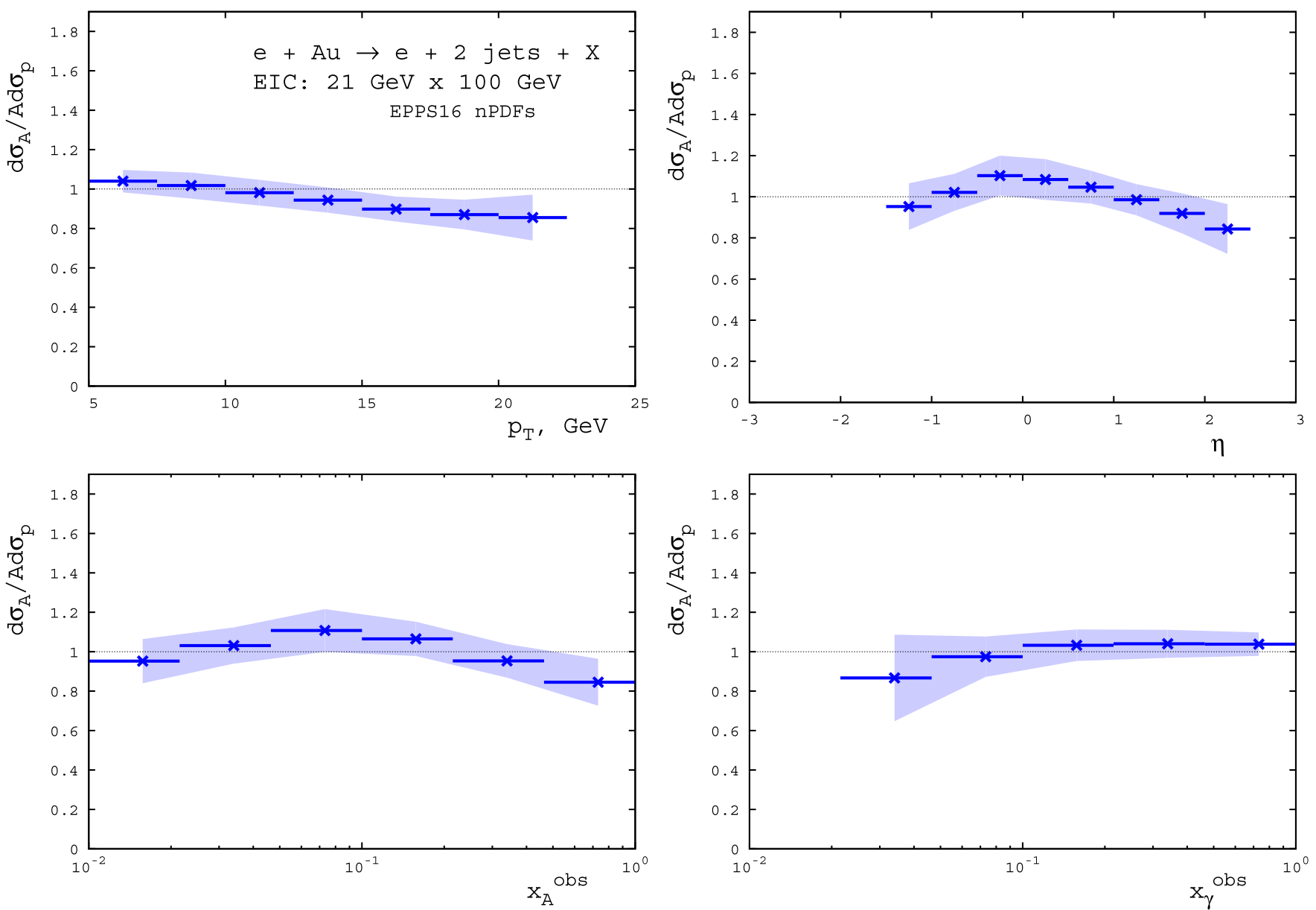

FIG. 3. NLO QCD predictions for the ratio of the cross sections of dijet photoproduction on nuclei and the proton as a function of $\bar{p}_{T}$, $\bar{\eta}, x_{A}^{\mathrm{obs}}$, and $x_{\gamma}^{\mathrm{obs}}$ in the EIC kinematics. The calculation uses central values of EPPS16 nPDFs (solid lines) and 40 sets of error PDFs (shaded band). 

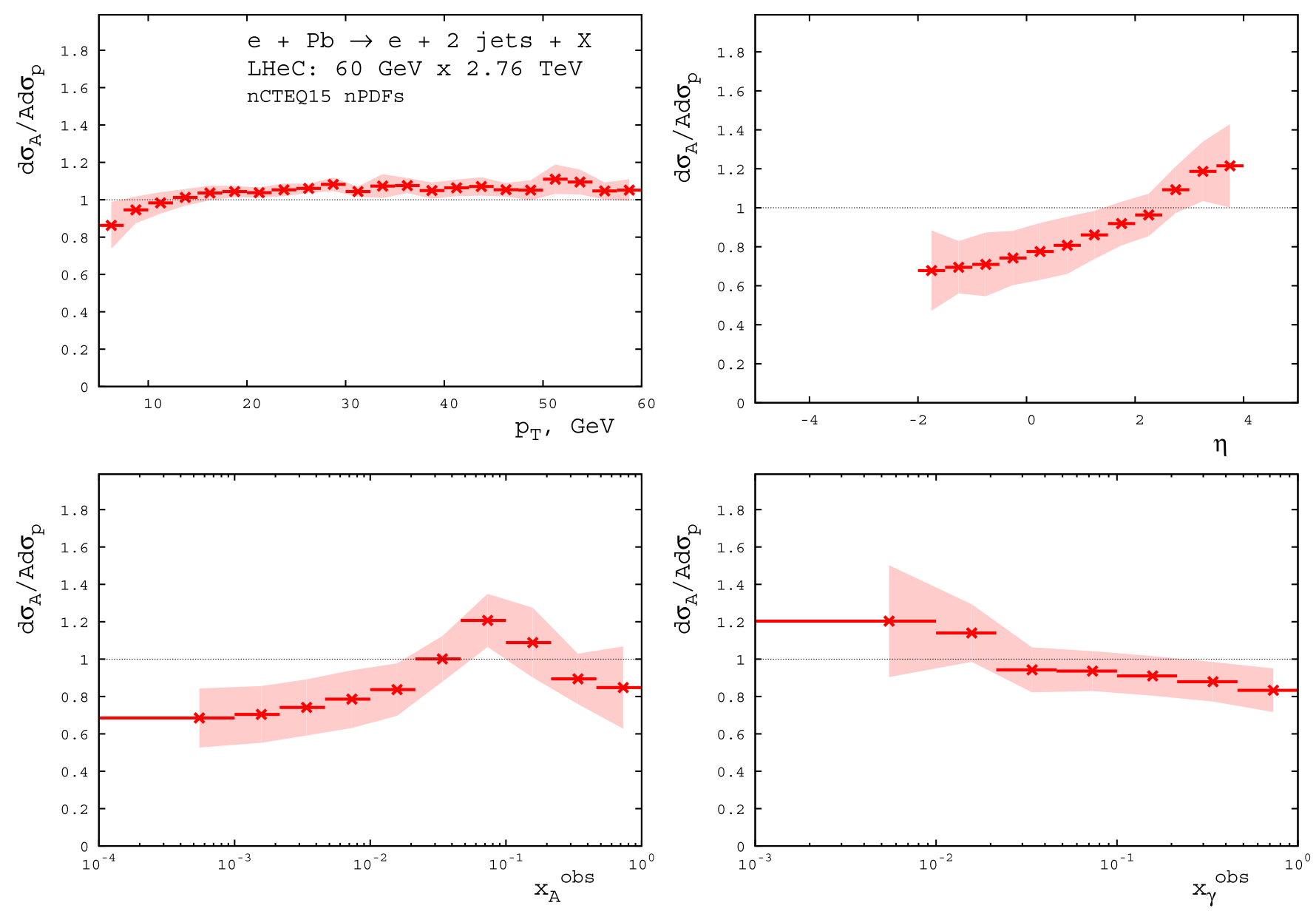

FIG. 4. Same as in Fig. 2 in the $\mathrm{LHeC}$ kinematics.

kinematics and in Figs. 4 and 5 in the LHeC kinematics. The results for the HE-LHeC and FCC closely resemble those for the $\mathrm{LHeC}$. The cross section ratios are shown as functions of $\bar{p}_{T}, \bar{\eta}, x_{A}^{\text {obs }}$, and $x_{\gamma}^{\text {obs }}$. In each bin, the solid lines correspond to the corresponding central value of nPDFs in the calculation of $d \sigma_{A}$ and $d \sigma_{p}$; the shaded band shows the theoretical uncertainty, which has been calculated using 32 nCTEQ15 error PDFs [18] and 40 EPPS16 error PDF sets [19].

In these figures, the results of the calculation using the central value of nPDFs exhibit a clear nuclear dependence of the presented distributions. At the EIC, the magnitude of nuclear modifications of the dijet cross section is of the order of $10-20 \%$ and is compatible to the theoretical uncertainty due to current uncertainties of nCTEQ15 and EPPS16 nPDFs. At the same time, nuclear modifications of $d \sigma_{A} /\left(A d \sigma_{p}\right)$ are more pronounced in the kinematics of LHeC (HE-LHeC, FCC) so that the predicted nuclear suppression of the $\bar{\eta}$ and $x_{A}^{\text {obs }}$ distributions is somewhat larger (in the nCTEQ15 case) than the uncertainty band due to nPDFs.

From the point of view of constraining nPDFs at small $x$, the distribution in $x_{A}^{\text {obs }}$ is the most important one. The shape of $d \sigma_{A} /\left(A d \sigma_{p}\right)$ repeats that of the ratio of the nucleus and proton structure functions $F_{2 A}\left(x, \mu^{2}\right) /\left[A F_{2 p}\left(x, \mu^{2}\right)\right]$ and parton distributions $f_{A}^{j}\left(x, \mu^{2}\right) /\left[A f_{p}^{j}\left(x, \mu^{2}\right)\right]$ (in particular, the ratio of the nucleus and proton gluon distributions): the nuclear suppression (shadowing) for $x_{A}^{\mathrm{obs}}<0.05$ is followed by some enhancement (antishadowing) around $x_{A}^{\mathrm{obs}} \approx 0.1$, which is then followed by the EMC-effect-like suppression for $x_{A}^{\text {obs }}>0.2$. While the EIC allows one to probe the dijet cross section down to $x_{A}^{\mathrm{obs}} \approx 0.01$, the LHeC extends the small- $x$ range down to $x_{A}^{\text {obs }} \approx 10^{-4}$ (down to $x_{A}^{\text {obs }} \approx 10^{-5}$ at FCC). It significantly enhances the sensitivity to nuclear modifications of nPDFs at small $x$.

An inspection of Figs. 1-5 allows one to qualitatively explain the obtained results. At the EIC, the dijet cross section is peaked around $x_{A}^{\mathrm{obs}} \approx 0.1$, where nPDFs are somewhat enhanced compared to the free proton case, and hence one expects that $d \sigma_{A} /\left(A d \sigma_{p}\right) \geqslant 1$ in the dominant part of the phase space, and in particular at small $\bar{p}_{T}$ and large $x_{\gamma}$. It also reveals the anticorrelation of $x_{\gamma}$ with $x_{A}: d \sigma_{A} /\left(A d \sigma_{p}\right)$ is simultaneously enhanced around $x_{A}^{\text {obs }} \approx 0.1$ (which corresponds to small $x_{A}$ in the EIC kinematics) and for large values of $x_{\gamma}^{\mathrm{obs}}$.

At the $\mathrm{LHeC}$, the dijet cross section is dominated by small $x_{A}, x_{A}^{\mathrm{obs}}<0.01$. Hence, one expects that the $d \sigma_{A} /\left(A d \sigma_{p}\right)$ cross-section ratio is suppressed in most of the phase space, which is indeed observed in Figs. 4 and 5. The anticorrelation of $x_{\gamma}$ with $x_{A}$ is also clearly seen: $d \sigma_{A} /\left(A d \sigma_{p}\right)<1$ for small $x_{A}^{\mathrm{obs}}$ and large $x_{\gamma}^{\mathrm{obs}}$. 

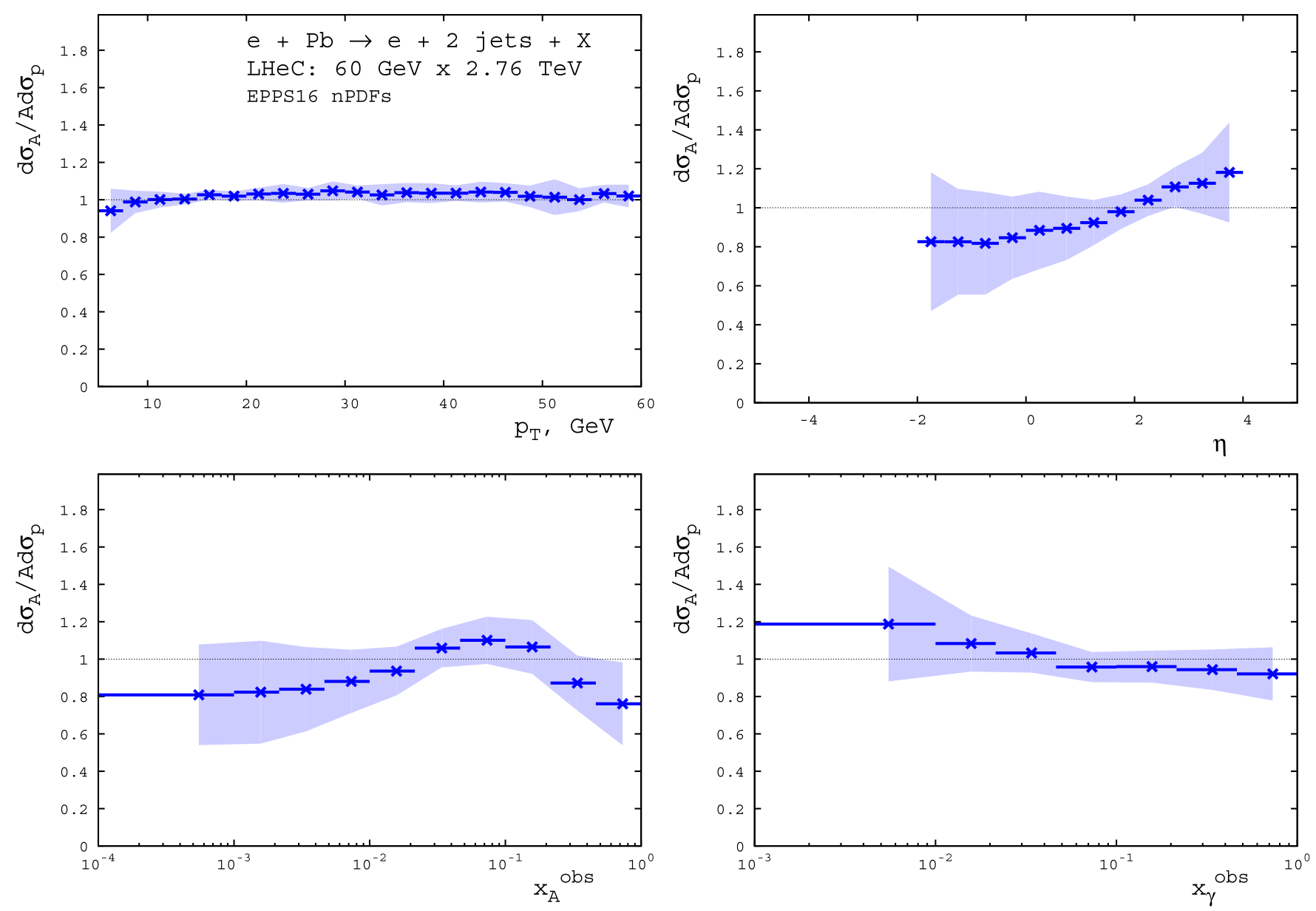

FIG. 5. Same as in Fig. 3 in the LHeC kinematics.

Note that the expected statistical uncertainty of measurements of the cross section of dijet photoproduction will be much smaller than the theoretical error bands due to nPDFs shown in Figs. 2-5. Indeed, using the projected integrated luminosity of $\int d t \mathcal{L}=10 \mathrm{fb}^{-1} /$ A for all four considered colliders $[5,8]$, one can readily estimate that the expected statistic uncertainty in each bin in Figs. 1-5 should be better than $1 \%$ . The expected systematic uncertainty is expected to be at the level of $2 \%$; see Refs. [8,20]. Hence, dijet photoproduction at future lepton-nucleus colliders can be used to considerably reduce the current uncertainties of nPDFs.

\section{CONCLUSIONS}

We calculated the cross section of inclusive dijet photoproduction in electron-nucleus scattering in the kinematics of such future lepton-nucleus colliders as EIC, LHeC, HE-LHeC, and FCC using NLO perturbative QCD and nCTEQ15 and EPPS16 nPDFs. We made predictions for the cross-section distributions as functions of the dijet average transverse momentum $\bar{p}_{T}$, the average rapidity $\bar{\eta}$, the nuclear momentum fraction $x_{A}^{\text {obs }}$, and the photon momentum fraction $x_{\gamma}^{\mathrm{obs}}$ and compared the kinematic reaches of the four colliders. We found that an increase of the collision energy from the EIC to the $\mathrm{LHeC}$ and beyond extends the coverage in all four considered variables. Notably, the LHeC and HE$\mathrm{LHeC}$ will allow one to probe the dijet cross section down to $x_{A}^{\text {obs }} \sim 10^{-4}$ (down to $x_{A}^{\text {obs }} \sim 10^{-5}$ at the FCC). We also calculated the ratio of the dijet cross sections on a nucleus and the proton, $\sigma_{A} /\left(A \sigma_{p}\right)$, and showed that it exhibits clear nuclear modifications. We found that in the important case of the $x_{A}^{\text {obs }}$ dependence, the shape of $\sigma_{A} /\left(A \sigma_{p}\right)$ repeats that of the ratio of the nucleus and proton parton distributions and, in particular, the $g_{A}\left(x, \mu^{2}\right) /\left[\operatorname{Ag}_{p}\left(x, \mu^{2}\right)\right]$ ratio, and reveals a strong suppression due to nuclear shadowing for $x_{A}^{\text {obs }}<0.01$. This indicates that dijet photoproduction in lepton-nucleus scattering in the kinematics of the future lepton-nucleus colliders will be very beneficial to reduce current uncertainties of nPDFs.

\section{ACKNOWLEDGMENTS}

M.K. would like to thank the Petersburg Nuclear Physics Institute (PNPI), Gatchina, for the kind hospitality extended to him during his research visit. V.G. would like to thank the Institut für Theoretische Physik, Westfälische Wilhelms-Universität Münster for hospitality. V.G.'s research is supported in part by RFBR, Research Project No. 17-52-12070. The authors gratefully acknowledge financial support of DFG through Grant No. KL 1266/9-1 
within the framework of the joint German-Russian project

"New constraints on nuclear parton distribution functions at small $x$ from dijet production in $\gamma A$ collisions at the LHC."
[1] M. Arneodo, A. Bialas, M. W. Krasny, T. Sloan, and M. Strikman, arXiv:hep-ph/9610423 [hep-ph].

[2] D. Boer, M. Diehl, R. Milner, R. Venugopalan, W. Vogelsang, D. Kaplan, H. Montgomery, S. Vigdor, A. Accardi, E. C. Aschenauer et al., arXiv:1108.1713 [nucl-th].

[3] A. Accardi et al., Eur. Phys. J. A 52, 268 (2016).

[4] J. L. Abelleira Fernandez et al. (LHeC Study Group), J. Phys. G 39, 075001 (2012).

[5] O. Brüning et al. (LHeC and PERLE Collaborations), J. Phys. G 46, 123001 (2019).

[6] A. Abada et al. (FCC Collaboration), Eur. Phys. J. C 79, 474 (2019).

[7] H. Paukkunen (LHeC study Group), PoS DIS2017, 109 (2018), arXiv: 1709.08342 [hep-ph].

[8] E. C. Aschenauer, S. Fazio, M. A. C. Lamont, H. Paukkunen, and P. Zurita, Phys. Rev. D 96, 114005 (2017).

[9] R. Abdul Khalek et al. (NNPDF), Eur. Phys. J. C 79, 471 (2019).

[10] J. J. Ethier and E. R. Nocera, Ann. Rev. Nucl. Part. Sci. 70, 43 (2020).

[11] C. Adloff et al. (H1 Collaboration), Eur. Phys. J. C 29, 497 (2003).

[12] H. Abramowicz et al. (ZEUS Collaboration), Nucl. Phys. B 864, 1 (2012).
[13] C. Adloff et al. (H1 Collaboration), Eur. Phys. J. C 25, 13 (2002).

[14] H. Abramowicz et al. (ZEUS Collaboration), Eur. Phys. J. C 70, 965 (2010).

[15] A. J. Baltz et al., Phys. Rept. 458, 1 (2008).

[16] V. Guzey and M. Klasen, Phys. Rev. C 99, 065202 (2019).

[17] V. Guzey and M. Klasen, Eur. Phys. J. C 79, 396 (2019).

[18] K. Kovarik et al., Phys. Rev. D 93, 085037 (2016).

[19] K. J. Eskola, P. Paakkinen, H. Paukkunen, and C. A. Salgado, Eur. Phys. J. C 77, 163 (2017).

[20] M. Klasen and K. Kovarik, Phys. Rev. D 97, 114013 (2018).

[21] M. Klasen and G. Kramer, Z. Phys. C 72, 107 (1996).

[22] M. Klasen and G. Kramer, Z. Phys. C 76, 67 (1997).

[23] M. Klasen, T. Kleinwort, and G. Kramer, Eur. Phys. J. D 1, 1 (1998).

[24] M. Klasen and G. Kramer, Eur. Phys. J. C 71, 1774 (2011).

[25] M. Klasen, Rev. Mod. Phys. 74, 1221 (2002).

[26] S. Frixione, M. L. Mangano, P. Nason, and G. Ridolfi, Phys. Lett. B 319, 339 (1993).

[27] M. Glück, E. Reya, and A. Vogt, Phys. Rev. D 46, 1973 (1992).

[28] M. Cacciari, G. P. Salam, and G. Soyez, J. High Energy Phys. 04 (2008) 063.

[29] M. Klasen and G. Kramer, Phys. Lett. B 366, 385 (1996). 